\title{
Impact of Leadership on Digital Transformation
}

\author{
Dr. Mouhamadou Sow \\ Faculty Member, School of Management, National American University \\ University of Phoenix, City University of Seattle, USA \\ Dr. Solomon Aborbie \\ Professor of Management Studies \\ Malone University, USA
}

Received: July 10, 2018 Accepted: July 24, 2018 Published: August 17, 2018

doi:10.5296/ber.v8i3.13368

URL: https://doi.org/10.5296/ber.v8i3.13368

\begin{abstract}
The purpose of this study was to identify leadership styles that impacted the digital transformation of an organization. Six information technology leaders from healthcare organizations were chosen to lead and participate in a digital transformation effort across several organizations in the Midwest (USA). The information collected was transcribed for clarity and to identify patterns in leadership styles that were evident. A qualitative research design using semi-structured interviews was established to determine this impact and how leadership style influenced an organization's direction regarding its digital transformation. Specific research literature in this area was addressed and provided a framework for this issue, including the types of leadership styles which were commonly used. These styles influenced large-scale decisions within an organization. In addition, the success of this transformation was evaluated in the context of leadership styles occurring naturally within an organization. The research findings indicated that leadership style was highly impactful in transforming organizations and employee-based involvement and input played a critical role in the success of the change effort. In addition, organizational leaders lacked some of the technical knowledge required to lead this project. Leadership styles are influential in organizations and can implement norms, expectations, and desirable outcomes during large-scale transformative projects.
\end{abstract}

Keywords: Digital transformation, Change management, Leadership style, Transactional leadership, Transformational leadership 


\section{Introduction}

When an organization seeks to transform from a manual process to a comprehensive digital platform, this requires a successful leadership strategy which can be influential and impactful in advancing this type of change over the long term. Many leaders do not have the technical knowledge or aptitude to oversee this type of transformation. However, their input is critical and requires the ability to be flexible. In addition, they must acquire new tasks and knowledge in the process or this change can lead to significant cost overruns and poor execution (Matt, Hess, \& Benlian, 2015). Organizations which employ leaders with different types of leadership styles may experience mixed results in this regard; therefore, the most effective type of leadership styles should be examined to determine how they impact decision-making during the process of digital transformation. Leaders should have some degree of influence and demonstrate their ability to adopt a strategy which will positively impact the digital transformation process rather than to limit its success over the long term (Allio, 2015). However, when the leadership style does not align with the digital transformation or when the strategy is ineffective, problems may emerge which could impact the organization in negative ways for many years to come (Allio, 2015). The purpose of the following study is to determine if differences in leadership styles are influential in the process of digital transformation within an organization and if this process can be further advanced in one direction or another due to leadership style.

The adoption of transformational, transactional, and other leadership styles could lead to successful results when a large-scale digital transformation is implemented. Furthermore, evidence regarding limited technical knowledge at the leadership level and its impact on the digital transformation must also be considered, as the appropriate strategy is necessary to ensure that the organization can be successful with its new technological capabilities and have the leaders in place to accomplish these goals effectively (Kane, Palmer, Phillips, Kiron, $\&$ Buckley, 2015). What remains to be seen is whether a leadership style has a direct impact on digital transformation or not, Therefore, the proposed research question is the following: What type of impact do different leadership styles have on digital transformation within an organization? This research question will be addressed in the following sections to determine if leadership styles can lead to the desired level of digital transformation among different organizations.

\section{Literature Review}

A literature review is designed to evaluate different types of research articles and studies which pertain to the topic in question and the research question(s) under consideration. In formulating any type of research study (qualitative or quantitative), the literature review offers evidence regarding the subject and research question(s). In addition, it identifies prior evidence if it exists to support this belief or assertion and if it may be applied in real-world settings. A literature review should be conducted by using a variety of databases which offer many different research articles through the selection of key words and phrases which narrow down the topic to identify literature of interest. Once specific articles are identified, they should be examined in conjunction with a proposed conceptual or theoretical framework 
which supports the research question(s) or hypotheses and other priorities. This framework guides the literature search and the selection of articles which provide relevant information regarding the research topic and evidence to support or negate the proposed claims. This process is essential to the discovery of new concepts or theories which influence the conclusions that are drawn and the potential to create impactful results for future studies. A literature review should not be meant to identify all possible articles and parameters regarding a research topic but should provide sufficient information to draw important conclusions and create new opportunities to advance research within the subject area. For this study, the conceptual framework evaluates Goleman's theory of emotional intelligence, which allows individuals to identify their abilities and skills and manage their emotions in different settings (Serrat, 2017). In addition, Berman's concepts related to digital transformation practices acknowledge that flexibility and customer responsiveness are critical to accommodate customer needs and improve an organization (2012). This is applicable to the study because organizations rely upon leaders who possess knowledge regarding digital transformation projects and have the emotional intelligence to recognize when their skills are limited in this focus area and allow others to take leadership over these issues.

Digital transformation practices require an organization to prepare for significant disruptions to their routine activities and processes, typically for a period of months to years, and these practices involve large-scale migration to data-driven technology-based systems, the acquisition of value for key stakeholders and customers, and a design which can effectively streamline processes, improve efficiency, and support sustainable activities to achieve growth and stability (Dahlstrom, Desmet, \& Singer, 2017). In this context, a digital transformation requires the following elements: identification of the future direction of the business; identification of leaders to direct the transformation; convincing key stakeholders that the transformation is a good idea; determining how the organization can be in a competitive position within the digital era; determining how decisions should be made during the transformation; obtaining funding to accomplish the goals of the transformation; and identifying areas where the organization can succeed with this effort and accomplish its goals effectively (Dahlstrom et al., 2017). A digital transformation requires significant human, financial, and technological capital, all of which must be aligned to facilitate successful results and must employ leaders who are capable of directing this change and allow their knowledge and experience to influence the process (Dahlstrom et al., 2017).

Demirkan, Spohrer, and Welser (2016) addressed the significance of the digital era and how organizations have evolved to accommodate the demands of their customers and key stakeholders. Specifically, digital transformation was noted as "the profound and accelerating transformation of business activities, processes, competencies, and models to fully leverage the changes and opportunities brought by digital technologies and their impact across society in a strategic and prioritized way" (Demirkan et al., 2016, p. 14). Based upon this definition, digital transformation requires significant organizational changes to take place and a shift in strategy which may be difficult to accomplish without a dramatic change in organizational culture and processes (Demirkan et al., 2016). This perspective supported the need for creative leaders who have had a significant impact on organizational decision-making, having 
progressive ideas, and who support "digital disruption" in ways which will lead to long-term benefits and capabilities for the organization (Demirkan et al., 2016). Organizations must have an e-leadership model in place to promote successful strategic change related to digital transformation and provide a level of much-needed stability during the change process $(\mathrm{Li}$, Liu, Belitski, Ghobadian, \& O’Regan, 2016). Any digital transformation requires a strategic model which will lead to a successful implementation and improved outcomes once the change is complete (Berman, 2012). In organizations with a largely customer-based focus, it is necessary to "capture the dynamic change from company ecosystem to customer ecosystem" with a new strategy designed to embrace what a digital transformation has to offer (Lipiainen, 2015). Digital leadership is essential to the success of any digital transformation and requires a focus on customer engagement, the need for advanced technical tools and a high capacity for storage, and a framing of the culture to support digitization in different forms (El Sawy, Kraemmergaard, Amsinck, \& Vinther, 2016).

Leadership styles are essential to an organization and can drive successful results; however, not all organizations employ the appropriate styles for their existing business practices, while others utilize leadership styles which align with organizational needs. When an organizational change is required, the appropriate leadership style may make this transition somewhat easier, such as the transformational and transactional styles to improve managerial engagement and enthusiasm regarding the change (Holten \& Brenner, 2015). In this context, transformational leaders can provide an example for others to follow, inspire others to be successful, develop a shared vision, and empower creativity (Holten \& Brenner, 2015). Some organizations may employ leaders who have differing leadership styles, such as transformational towards motivating others and creativity, while transactional leaders are largely focused on setting the tone for others to follow without room for innovation (Baskarada, Watson, \& Cromarty, 2016). These differences must be balanced in such a way that they do not distract from organizational priorities and provide a leadership framework which will offer positive results during the change process (Baskarada et al., 2016). Similarly, participative leadership can provide employees with decision-making capabilities and influence positive outcomes as needed for a successful change management strategy (Fatima, Majeed, \& Saeed, 2017). Furthermore, innovative (participative) leadership must be part of this process and provide inspiration to employees to embrace new forms of knowledge and promote greater creativity to implement strategic change (Tung \& Yu, 2016).

Cultural dynamics play a critical role in the success of a digital transformation and provide a framework for change; therefore, change management strategies must be aligned with these priorities and the practices currently taking place and those which will be desirable in the future (Kasemsap, 2015). In addition, "culture is a tool to encourage commitment and achieve the goals of an organization" (Kasemsap, 2015, p. 297). Under these circumstances, the organizational culture drives change, including digital transformation; therefore, the organizational culture should set the tone for this type of change and secure support from leaders and employees to ensure that the change is successful (Kasemsap, 2015).

A digital transformation requires a significant shift in processes and procedures within an organization and necessitates that employees understand how this change may impact their 
roles and responsibilities (Vey, Fandel-Meyer, Zipp, \& Schneider, 2017). It is important for an organization's core leadership to support this type of change and to have the vision that is required to accomplish related goals and objectives, even if the technical knowledge is not readily available (Vey et al., 2017). In this capacity, it is important for leaders to accomplish the following: "Handling such a fundamental change requires lots of imagination. Assume a company's leaders fully recognize the current development and can even imagine a vision to react to it" (Vey et al., 2017, p. 25). These conditions require leaders who can embrace change and can inspire others to view the change in a positive light (Vey et al., 2017), such as transformative leaders with a vision for the future which includes employee empowerment and creativity among other characteristics. At the same time, antithetic leadership is a term which should be considered because it combines the concepts of business (operations and transformation) with design (innovation and idea development), both of which are essential to a successful digital transformation within an organization (von Kutzschebach, Mittemeyer, \& Wagner, 2017). These differences are significant and may cause unwanted conflicts when leaders do not necessarily understand or embrace the technical requirements of digital transformation and may lead to setbacks or bottlenecks which must be worked through (von Kutzschebach et al., 2017). Due to these realities, organizations with leaders who are willing to embrace change and can empower others through this process may achieve higher levels of success during a digital transformation and may experience greater stability, particularly when the organizational culture is built to withstand tension and tumultuous circumstances (von Kutzschebach et al., 2017). It is imperative that organizations which undergo a digital transformation should have the appropriate leaders in place to maintain stability and secure successful results throughout the change and after the transformation is complete.

Within an organization, preparing for a digital transformation requires the appropriate technical knowledge and the ability to withstand disruptions during this process (Kiron, Kane, Palmer, Phillips, \& Buckley, 2016). It is known that "The main characteristics of digital cultures include: an expanded appetite for risk, rapid experimentation, heavy investment in talent, and recruiting and developing leaders who excel at "soft" skills. Leading a digital company does not require technologists at the helm" (Kiron et al., 2016, pp. 2-3). However, leaders must be willing to allow others to lead in the technical areas and limit their control by developing a culture of trust which empowers others to succeed and utilize their knowledge towards successful outcomes (Kiron et al., 2016). In this context, leaders must utilize their strengths wisely and allow others to lead in areas where their weaknesses prevail to ensure that the organization is fully prepared to implement the digital transformation with the best interests of the culture, employees, and key stakeholders in mind.

Some organizations may have leaders who are skeptical of any large-scale changes. They operate with a largely traditional and bureaucratic framework where change is not desirable. With digital transformation and related practices, the skepticism regarding these changes may be very high and limit the potential for a successful implementation (Kolbjornsrud, Amico, \& Thomas 2017). Therefore, training and education regarding the benefits of these practices must be provided to leaders and allow organizations to take the next steps forward to overcome their limitations and to embrace a new strategy that is likely to have lasting 
benefits (Kolbjornsrud et al., 2017). When digital transformation is imminent, technical knowledge is essential but should be aligned with organizational priorities rather than in another direction which could lead to strategic failure (Gerth \& Peppard, 2015).

\section{Method}

This qualitative research study was phenomenological in nature and was based upon an interview method to evaluate leaders involved in digital transformations to evaluate the significance and impact of leadership style on this process. This was the preferred method because understanding how leaders perceive or are involved in digital transformations required expression of their views of this phenomenon. The interview method also enabled leaders to share their preferred leadership styles as a means of determining if these approaches impact digital transformation practices. As part of these interviews, common leadership styles such as transformative and transactional were considered to determine if this approach influenced the success or contributes to setbacks or complications for the leaders who were interviewed. In addition, when technical knowledge is limited within the leadership realm, the degree of impact of this phenomenon was necessary because it could contribute to poor strategic decision-making or misconceptions regarding the large-scale impact of the digital transformation. This research method was preferred because it provided direct insight into the leader's role in digital transformation and how leaders from healthcare organizations perceived their contributions from a personal perspective.

The research methodology emphasized the importance of digital transformation and the digital age and applied leadership approaches to determine its effectiveness in modern organizations. Some of the most important factors considered included the organizational culture, the concept of emotional intelligence as it was proposed by Goleman (2006), and other common leadership theories which influenced decision-making and approaches to strategic development within organizations (Leban \& Zulauf, 2004). A successful research discussion utilizes a qualitative approach to determine the extent to which digital transformation was impacted by leadership styles, and interviews provided insight regarding this transformation across different areas.

The selection of interviews was based upon the perceptions of leaders and their express knowledge of digital transformation practices. Even when leaders lack specific technical knowledge, they played prominent roles because of their leadership characteristics and input. This was important to the selection of prospective participants for the interviews to ensure that leaders with different leadership styles and knowledge of technical matters were selected. During the selection process, groups of leaders were considered who possessed various levels of experience with technological implementations, including some who had no direct experience with these practices but nonetheless held strong views regarding these practices. Leaders were identified from a group of organizations who had undergone or were in the process of undergoing a digital transformation and had strong views regarding this process. Based upon this group, individual leaders were randomly selected for interviews. Specifically, six interviews were conducted individually for this study, 30-45 minutes each in length with a set of pre-determined questions and additional time for other responses and feedback. A 
smaller group of participants was warranted and was selected on purpose because it enabled the interviewees to provide lengthier responses and to address the issues in greater detail. This process was comprised of a semi-structured interview format to ensure that participants answered the pre-determined questions but could also provide additional information that was not part of the structured format. Since the interviews were recorded, the researchers transcribed the data to identify patterns among the responses, along with other data which did not fit any specific pattern. This data was important in determining the level of experience of each leader with a digital transformation, the type of leadership styles that were employed, and other information which was relevant to the study results.

\section{Results}

The results of the interviews indicated that although leadership played a critical role in the implementation of any large-scale organizational change such as a digital transformation, there was not one specific leadership style that has an optimal positive impact. It was determined that some leaders were resistant to change and found it difficult to embrace a digital transformation, particularly if they lacked sufficient technical knowledge to understand the scope of the change and its impact on the organization. In addition, this implementation was difficult for some leaders to give up some of the control and to allow those with technical knowledge to take over some of the implementation. It was also determined that a transformational leadership style was at least partially conducive to the desired outcomes during a digital transformation, but other leadership styles had influence on this process. Leaders indicated that the change management strategy should be aligned with the organizational culture in any situation and employees should be provided with the necessary training, knowledge, support, and guidance to embrace the change more effectively.

\section{Discussion}

A digital transformation strategy necessitated the collaboration of leaders, resources, and teams to ensure that an implementation was successful and did not significantly disrupt operations. However, this does not appear to be aligned to one specific type of leadership style, based upon existing research and the interviews that were conducted. It was believed that transformative leaders have had greater impact on employees' motivation and creativity to succeed during this type of change, but it was not the sole means by which an organization achieved a successful implementation. Other factors and leadership characteristics had a significant impact during a digital transformation. Most importantly, in the future, organizational leaders must participate in a strong organizational culture which embraces change but also provides a level of stability that will have a lasting impact on employees and teams. The study recognized that key characteristics were essential to achieve success with a digital transformation, such as emotional intelligence, motivation, and empowerment. However, additional research is necessary to further distinguish among these principles and the leadership styles which lead to successful results during digital transformation.

Organizational leadership must demonstrate the willingness to change and to motivate others to support the change effort while also remaining receptive and recognizing that a digital 
transformation may not cater to their key strengths, particularly if they lack the technical knowledge to implement many of the steps involved in the change. To determine if different leadership styles directly impact digital transformation, additional research is necessary in this subject area. The research should consider organizations which have implemented this type of strategic change and an evaluation of the leadership styles which have been utilized. For instance, transformational leadership and emotional intelligence may align to support inspirational leadership that will translate into successful results (Leban \& Zulauf, 2004).

\section{Conclusion}

Organizations which undergo a digital transformation must have the tools available to implement this large-scale change, including the technical knowledge to ensure that its outcomes are successful. Organizational leaders must demonstrate a critical understanding of this process and be willing to embrace change, along with understanding that when they align their leadership characteristics with the change management strategy, it is likely to be more effective. A variety of leadership characteristics are necessary to ensure that the change can be accomplished, and these may not align with a specific leadership style. At the same time, leaders must develop an acceptable level of trust in their employees and other managers who have specific knowledge regarding this type of change so that creativity and innovation can thrive in this setting. It is imperative that organizations have leaders who can adapt to change effectively, can support a diverse organizational culture, and have the patience and wherewithal to withstand disruptions and other challenges during the implementation phase. The interviews conducted for this qualitative study indicated that the outcomes of this change are contingent upon a strategy which supports realistic and practical results and empowers employees to make contributions that will best utilize their core strengths in the process. Therefore, leaders must be flexible and support their employees who can make a difference during and after a digital transformation, along with recognizing what they have to offer and how they can make a difference at the organization in the long term.

\section{References}

Allio, R. J. (2015). Good strategy makes good leaders. Strategy \& Leadership, 43(5), 3-9. https://doi.org/10.1108/SL-07-2015-0059

Baškarada, S., Watson, J., \& Cromarty, J. (2017). Balancing transactional and transformational leadership. International Journal of Organizational Analysis, 25(3), 506-515. https://doi.org/10.1108/IJOA-02-2016-0978

Berman, S. J. (2012). Digital transformation: Opportunities to create new business models. Strategy \& Leadership, 40(2), 16-24. https://doi.org/10.1108/10878571211209314

Dahlström, P., Desmet, D., \& Singer, M. (2017). The seven decisions that matter in a digital transformation: a CEO's guide to reinvention. Digital McKinsey article (Feb 2017).

Demirkan, H., Spohrer, J. C., \& Welser, J. J. (2016). Digital innovation and strategic transformation. IT Professional, 18(6), 14-18. https://doi.org/10.1109/MITP.2016.115

El Sawy, O. A., Kræmmergaard, P., Amsinck, H., \& Vinther, A. L. (2016). How LEGO Built 
the Foundations and Enterprise Capabilities for Digital Leadership. MIS Quarterly Executive, 15(2).

Fatima, T., Majeed, M., \& Saeed, I. (2017). Does Participative Leadership Promote Innovative Work Behavior: The Moderated Mediation Model. Business \& Economic Review, 9(4), 139-156. https://doi.org/10.22547/BER/9.4.7

Gerth, A. B., \& Peppard, J. (2016). The dynamics of CIO derailment: How CIOs come undone and how to avoid it. Business Horizons, 59(1), 61-70.

https://doi.org/10.1016/j.bushor.2015.09.001

Goleman, D. (2006). Emotional intelligence. New York: Bantam.

Holten, A. L., \& Brenner, S. O. (2015). Leadership style and the process of organizational change. Leadership \& Organization Development Journal, 36(1), 2-16.

https://doi.org/10.1108/LODJ-11-2012-0155

Kane, G. C., Palmer, D., Phillips, A. N., Kiron, D., \& Buckley, N. (2015). Strategy, not technology, drives digital transformation. MIT Sloan Management Review and Deloitte University Press, 14. Retrieved from

https://sloanreview.mit.edu/projects/strategy-drives-digital-transformation/

Kasemsap, K. (2017). The role of cultural dynamics in the digital age. In Organizational Culture and Behavior: Concepts, Methodologies, Tools, and Applications (pp. 1144-1162). IGI Global. https://doi.org/10.4018/978-1-5225-1913-3.ch054

Kiron, D., Kane, G. C., Palmer, D., Phillips, A. N., \& Buckley, N. (2016). Aligning the organization for its digital future. MIT Sloan Management Review, 58(1). Retrieved from http://pedrotrillo.com/wp-content/uploads/2016/01/58180-MITSMR-Deloitte-Digital-Busines s-2016.pdf

Kolbjørnsrud, V., Amico, R., \& Thomas, R. J. (2017). Partnering with AI: how organizations can win over skeptical managers. Strategy \& Leadership, 45(1), 37-43.

https://doi.org/10.1108/SL-12-2016-0085

Leban, W., \& Zulauf, C. (2004). Linking emotional intelligence abilities and transformational leadership styles. Leadership \& Organization Development Journal, 25(7), 554-564.

https://doi.org/10.1108/01437730410561440

Li, W., Liu, K., Belitski, M., Ghobadian, A., \& O'Regan, N. (2016). e-Leadership through strategic alignment: an empirical study of small-and medium-sized enterprises in the digital age. Journal of Information Technology, 31(2), 185-206. https://doi.org/10.1057/jit.2016.10

Lipiäinen, H. S. M. (2015). CRM in the digital age: implementation of CRM in three contemporary B2B firms. Journal of Systems and Information Technology, 17(1), 2-19. https://doi.org/10.1108/JSIT-06-2014-0044

Matt, C., Hess, T., \& Benlian, A. (2015). Digital transformation strategies. Business \& Information Systems Engineering, 57(5), 339-343. 
https://doi.org/10.1007/s12599-015-0401-5

Serrat, O. (2017). Knowledge Solutions. Singapore: Springer.

https://doi.org/10.1007/978-981-10-0983-9

Tung, F. C., \& Yu, T. W. (2016). Does innovation leadership enhance creativity in high-tech industries?. Leadership \& Organization Development Journal,37(5), 579-592. https://doi.org/10.1108/LODJ-09-2014-0170

Vey, K., Fandel-Meyer, T., Zipp, J. S., \& Schneider, C. (2017). Learning \& Development in Times of Digital Transformation: Facilitating a Culture of Change and Innovation. International Journal of Advanced Corporate Learning (iJAC), 10(1), 22-32. https://doi.org/10.3991/ijac.v10i1.6334

Von Kutzschenbach, M., Mittemeyer, P., \& Wagner, W. (2017). Antithetic Leadership: Designers Are Different, Business People Too. In Shaping the Digital Enterprise (pp. 93-107). Springer, Cham. https://doi.org/10.1007/978-3-319-40967-2_4

\section{Copyright Disclaimer}

Copyright for this article is retained by the author(s), with first publication rights granted to the journal.

This is an open-access article distributed under the terms and conditions of the Creative Commons Attribution license (http://creativecommons.org/licenses/by/3.0/). 